\title{
Extração de Terceiros Molares Retidos sob Anestesia Local. Avaliação de Ansiedade, Dor, Alterações Hemodinâmicas e Respiratórias.
} Extraction of Retained Third Molar Teeth under Local Anesthesia. Evaluation of Anxiety, Pain, Hemodynamic and Respiratory Alterations.

\section{Angelica de Fátima de Assunção Braga ${ }^{1}$, Laura Helena D’Ottaviano}

\section{Abstract}

A transversal, descriptive study with an analytical component was conducted, aimed at evaluating anxiety during the period preceding the procedure, as well as hemodynamic and respiratory alterations and pain during the intraoperative period in patients undergoing the extraction of retained third molars under local anesthesia. Forty-two healthy male subjects, ranging in age from 18 to 34 years were included in the study. Anesthesia was performed with lidocaine hydrochloride $2 \%$ with epinephrine $1: 50.000$, at a dose ranging from 72 to $144 \mathrm{mg}$. A non-invasive multiparametric monitor was used to monitor the following parameters: mean arterial blood pressure, heart rate, peripheral $\mathrm{O}_{2}$ saturation, respiratory rate; level of anxiety (anxiety scale for dental surgery) and pain (visual analog scale). Statistical analysis was performed with a paired Student's $t$ and Wilcoxon's test. The significance level was set at $5 \%$. In this study, twenty patients had some level of anxiety. Severe pain was reported in seven patients who required supplementation with local anesthesia during osteotomy, tooth sectioning and luxation. Individual analysis of hemodynamic and respiratory parameters showed alterations that were considered significant. The results of this study enabled us to conclude that hemodynamic and respiratory alterations may occur during the extraction of retained third molars. Monitoring is important for the detection and thus prevention of these complications. Furthermore, it is particularly useful in patients in whom these repercussions must be avoided.

Keywords: Dental surgery, Third molars, Local anesthesia, Hemodynamic and respiratory alterations, Monitoring.

\section{Resumo}

Trata-se de estudo transversal, descritivo com componente analítico, com o objetivo de avaliar a ansiedade no período que antecede 0 procedimento, as alterações hemodinâmicas, respiratórias e dor no intra-operatório em pacientes submetidos à extração de terceiros molares retidos sob anestesia local. Foram incluídos no estudo 42 sujeitos do gênero masculino, hígidos, com idade compreendida entre 18 e 34 anos. A anestesia foi feita com cloridrato de lidocaína a $2 \%$ com epinefrina 1:50.000, na dose que variou de 72 a 144mg. Com auxilio de monitor multiparamétrico não invasivo avaliaram-se os parâmetros: pressão arterial média, frequencia cardíaca, saturação periférica de $\mathrm{O}_{2}$ e frequencia respiratória; o grau de ansiedade (escala de ansiedade para cirurgia odontológica) e dor (escala analógica visual). A análise estatística foi realizada utilizando os testes $T$ de Student pareado e Wilcoxon pareado. O nível de significância foi de $5 \%$.

Neste estudo, vinte pacientes apresentavam algum grau de ansiedade. Dor intensa foi referida por sete pacientes nos momentos da osteotomia, odontosecção e luxação que necessitaram de complementação com anestésico local. A análise individual dos parâmetros hemodinâmicos e respiratórios mostrou alterações
${ }^{2}$, Franklin Sarmento da Silva Braga $^{3}$, Sirlei Siani Morais ${ }^{4}$

1 Professora Livre Docente do Departamento de Anestesiologia - Faculdade de Ciências Médicas da UNICAMP - Campinas - SP - Brasil

2 Mestre em Cirurgia - Serviço de Odontologia do Hospital das Clínicas da UNICAMP - Campinas - SP - Brasil

${ }^{3}$ Professor Doutor do Departamento de Anestesiologia - Faculdade de Ciências Médicas da UNICAMP - Campinas - SP - Brasil

${ }^{4}$ Estatística da Comissão de Pesquisa do CAISM - UNICAMP - Campinas - SP Brasil

Correspondência: Angelica de Fátima de Assunção

Endereço: Av. Dr. José Bonifácio Coutinho Nogueira, 225 - Torre D - Apto 61 Jardim Madalena, CEP 13091-611, Campinas - SP, Brasil

Data de Submissão: 02/05/2011

Data de Aceite: 07/11/2011

consideradas significativas. Os resultados deste estudo permitem concluir que alterações hemodinâmicas e respiratórias podem ocorrer durante extrações de terceiros molares retidos. A monitorização é importante na detecção e consequentemente na prevenção dessas intercorrências, sendo particularmente útil em pacientes em que essas repercussões devam ser evitadas.

Palavras-chave: Cirurgia dental, Terceiros molares, Anestesia local, Alterações hemodinâmicas e respiratórias, Monitorização.

\section{Introdução}

Procedimentos odontológicos, particularmente a extração de terceiros molares retidos, podem se tornar extremamente incômodos e dolorosos, gerando ansiedade, medo e até terror. Embora técnicas eficientes de anestesia local sejam utilizadas rotineiramente pelos cirurgiões dentistas, não é raro encontrarmos pacientes com fobia ao tratamento odontológico, sendo este sentimento causa de importantes alterações autonômicas (BRAND et al., 1995; BRAND; ABRAHAM-INPIJN, 1996).

Essas alterações podem se manifestar clinicamente no intraoperatório por repercussões cardiocirculatórias e respiratórias que justificam sobremaneira 0 emprego de métodos de monitorização. Particularmente, está indicada durante a extração cirúrgica de molares, em procedimentos traumáticos, ou com 0 objetivo de proporcionar maior segurança em pacientes emocionalmente comprometidos. Além de aumentar a margem de segurança do paciente durante a cirurgia, permite ao cirurgião dentista identificar imediatamente situações de risco aumentado, estabelecer precocemente um diagnóstico e prevenir possíveis complicações (BRAND et al., 1995; BRAND; ABRAHAM-INPIJN, 1996; ARRIGONI; LAMBRECTH; FILIPPI, 2005).

O emprego de equipamentos eletrônicos para a monitorização de dados vitais é muito mais eficiente e sensível do que os métodos tradicionais realizados através de observação visual da cor da pele, permeabilidade de vias aéreas, movimentação torácica, ausculta pulmonar e cardíaca com auxílio de estetoscópio precordial (CROSWELL et al., 1995; POLIDO; OLIVEIRA, 1998). 
Estudo de revisão realizado para avaliar presença de intercorrências durante procedimentos realizados em consultórios odontológicos demonstrou que $62 \%$ dos pacientes estavam sendo monitorados apenas através de observação visual. Estatísticas mostram que a morbidade e a mortalidade neste tipo de procedimento resultam de falha na conduta profissional, especialmente na subutilização de monitoramento e manobras de ressuscitação (CROSWELL et al., 1995).

Este estudo realizado em consultório odontológico, teve por objetivo avaliar em pacientes submetidos à extração de terceiros molares retidos sob anestesia local, as repercussões hemodinâmicas e respiratórias, a dor referida pelo paciente no momento do procedimento, bem como o grau de ansiedade no período que antecede o procedimento cirúrgico.

\section{Materiais e Métodos}

Trata-se de estudo transversal, prospectivo, descritivo, com componente analítico. O tamanho da amostra foi calculado para avaliar a diferença na variável dependente saturação periférica de $\mathrm{O}_{2}$ $\left(\mathrm{SpO}_{2}-\%\right)$, entre o início da cirurgia e o momento até completar a extração, utilizando dados de ALEMANY-MARTINEZ et al. (2008), teste t pareado, erro tipo alfa $=5 \%$ e um poder de $80 \%$, resultando em $\mathrm{n}=40$ pacientes.

Após aprovação pelo Comitê de Ética da Faculdade de

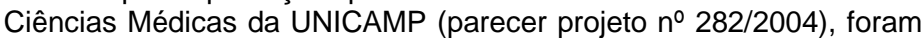
incluídos no estudo 42 pacientes do gênero masculino, estado físico I pela Classificação da American Society of Anesthesiologists (ASA), programados para cirurgia eletiva de extração de terceiros molares retidos, sob anestesia local, atendidos em consultório odontológico do serviço de Odontologia do Hospital de Clínicas da Unicamp e que aceitaram voluntariamente participar da pesquisa.

Constituíram critérios de exclusão: gênero feminino, portadores de doença sistêmica ou psiquiátrica, história de hipersensibilidade a anestésicos locais, fumantes e índice de massa corpórea (IMC) maior do que 30.

No momento da admissão do paciente no consultório odontológico, foi aplicada a Escala de Ansiedade preconizada por Corah et al. para pacientes submetidos a procedimentos odontológicos (Quadro 1). A escala é composta de 4 questões, cada uma com cinco alternativas, pontuadas de 1 (a) a 5 (e). O grau de ansiedade foi classificado de acordo com pontuação obtida da soma dos escores das quatro questões em: calmo (escore de 4 a 8); pouco tenso (escore de 8 a 10); tenso (escore de 11 a 12); ansioso (escore de 13 a 14) e muito ansioso (escore igual ou maior do que 15).

Nenhum paciente estava em jejum de mais de 3 horas, ou recebeu qualquer tipo de medicação pré-operatória. Foram monitorizados continuamente para obtenção de dados vitais, com monitor multiparamétrico - DIXTAL Modelo DX 2010 (cardioscópio, oxímetro de pulso e monitor não invasivo de pressão arterial). A temperatura ambiente foi mantida acima de $20^{\circ} \mathrm{C}$, e foram observadas as mãos do paciente, mantendo-as aquecidas antes e durante o procedimento, para que não houvesse alteração da perfusão periférica. A cadeira odontológica foi posicionada com o encosto reclinado entre 150 e 170 graus com relação ao assento, permanecendo nesta posição durante todo o procedimento.

Foram utilizadas técnicas anestésicas rotineiramente empregadas para extração de terceiros molares. Para os dentes superiores realizou-se a técnica de infiltração em vestíbulo bucal, em fundo de sulco gengivo-geniano, complementada por infiltração em mucosa palatina, em região de túber da maxila. Para os dentes inferiores utilizou-se a anestesia regional pterigomandibular (troncular), com bloqueio nos nervos alveolar inferior e nervo lingual, complementada por infiltração em vestíbulo mandibular, em região de primeiro molar, para anestesia do nervo bucinador. O anestésico local empregado foi o cloridrato de lidocaína a 2\% com epinefrina 1:50.000, na dose que variou de 72 a 144mg, correspondente ao conteúdo de 2 a 4 tubetes $(1,8 \mathrm{ml} /$ tubete $)$.

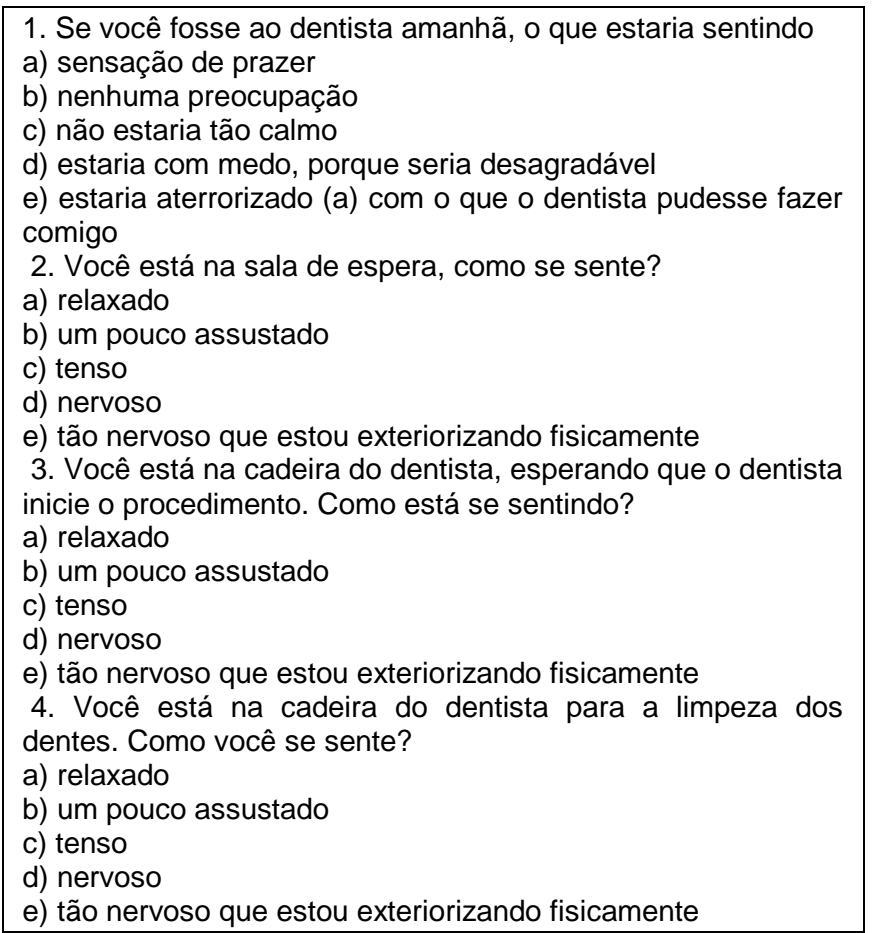
Quadro 1- Escala de Ansiedade para Procedimentos Dentários (CORAH; GALE; ILLIG, 1978)

Depois da anestesia local, para a extração dos dentes superiores e inferiores, foi realizada incisão intraoral de Mead, divulsão e descolamento dos tecidos moles, exposição do osso alveolar, osteotomia através de caneta de alta rotação e broca cirúrgica do tipo Zekrya, odontosecção com a mesma broca cirúrgica (quando necessário), avulsão dentária, curetagem do alvéolo, irrigação com soro fisiológico e sutura por pontos separados, com agulha triangular e fio de seda 4-0.

Foram avaliados no intraoperatório os seguintes parâmetros:1) saturação periférica de oxigênio $\left(\mathrm{SpO}_{2}\right)$ de acordo com metodologia proposta por BRAZ, 1996: $\geq 95 \%$ (saturação normal), 9490 (hipoxemia leve), 89-86 (hipoxemia moderada), < 86 (hipoxemia grave); 2) pressão arterial média $(\mathrm{mmHg})$; 3) frequência cardíaca (bpm); 4) frequência respiratória (rpm) - número de excursões torácicas por minuto; 5) Dor durante o procedimento com auxílio da Escala Análogo-Visual - EAV. A avaliação dos escores de dor de 0 a 10 foram estratificados em 3 níveis, a fim de aproximar a classificação a leve, moderada e intensa, portanto dividiu-se o valor máximo (10) por $3(3,33)$ - sendo formados os intervalos com variação de 3 em 3 pontos: 1 a 3,4 a 6 e > 6 . Antes do início do procedimento, a EAV era apresentada e explicada aos sujeitos da pesquisa, como deveriam pontuar na escala a intensidade da dor, assim como 0 momento do procedimento em que ocorria.

Os parâmetros hemodinâmicos e respiratórios foram analisados nos seguintes momentos: admissão no consultório odontológico (controle), imediatamente após a aplicação da anestesia 
local, a cada cinco minutos, durante todo o ato cirúrgico, e cinco minutos após o término do procedimento.

Os resultados foram expressos em médias e desviospadrão, frequências absolutas (n) e relativas (\%). Para avaliar a modificação dos parâmetros ao longo do tempo (inicial até 60 minutos), preferencialmente deve-se utilizar ANOVA para medidas repetidas. No entanto, esta análise somente levará em consideração os sujeitos sem perda de seguimento, isto é, requer que existam dados em todos os pontos a serem avaliados ao longo do tempo. Neste tipo de estudo, como cada sujeito possui um tempo de cirurgia diferente, observou-se que aos 35 minutos, mais de $20 \%$ dos sujeitos já haviam encerrado o procedimento. A aplicação de ANOVA para medidas repetidas, até os 60 minutos, seria executável em apenas $n$ = 12 sujeitos. Além disso, os tempos finais de cada procedimento são diferentes para cada sujeito (já que o tempo total de cirurgia é variável). A fim de minimizar os efeitos destes tipos de vieses (inerentes ao procedimento), para as variáveis dependentes, em cada um dos momentos, foi calculada a variação percentual em relação ao momento inicial: $100 \mathrm{X}$ (valor no momento i - valor inicial) / valor inicial, onde $i=5,10,15,20,25,30,35,40,45,50,55$ e 60 minutos. Esta medida também nos traz a vantagem de eliminar 0 viés de cada sujeito partir de um valor diferente no início do procedimento. Baseado nesta medida, foram descritos os valores médios e desviospadrão e aplicado os testes de Kolomogorv-Smirnov para avaliar a aderência à distribuição normal de cada uma das variáveis. Para avaliar a diferença em relação ao inicial, quando houve distribuição normal, utilizou-se o teste t de Student pareado, ou o correspondente teste não-paramétrico (teste de Wilcoxon pareado) para distribuição não-normal. O nível de significância foi de $5 \%(p<0,05)$, e o software utilizado para análise foi o SAS versão 8.2.

\section{Resultados}

Os valores médios, desvios padrão, valores mínimos e máximos de idade, peso, índice de massa corpóreo e tempo de cirurgia, encontram-se na Tabela 1.

Tabela 1- Características da Amostra

\begin{tabular}{l|cccc}
\hline \hline & Média & $\begin{array}{c}\text { Desvio } \\
\text { padrão }\end{array}$ & Mínima & Máxima \\
\hline \hline Idade (anos) & 22,9 & 4,4 & 18 & 34 \\
Peso $(\mathrm{kg})$ & 69,4 & 10 & 53 & 100 \\
IMC $(\mathrm{kg} / \mathrm{m} 2)$ & 22,8 & 2,4 & 18,5 & 27,4 \\
Tempo & 48,6 & 24,5 & 10 & 101 \\
cirúrgico & & & & \\
(min) & & & & \\
\hline \hline
\end{tabular}

Em 73,8\% (31 pacientes), o tempo máximo de cirurgia foi de 60 minutos, considerado o tempo para análise estatística das variáveis dependentes. Na maioria dos tempos estudados, não foram observadas diferenças significativas nos valores médios da frequência cardíaca em relação aos do valor inicial, exceto aos 5 $(p=0,0047)$ e aos 15 minutos $(p=0,0038)$ após a realização da anestesia. Observou-se que a variação percentual média não ultrapassou $10 \%$ em relação ao valor inicial (Tabela 2). A análise individual dos casos mostrou que em 18 pacientes (42,9\%), pelo menos em algum momento dentre os 60 minutos, ocorreu uma variação acima de $20 \%$ da freqüência cardíaca (dados não mostrados).
Tabela 2- Valores médios e desvios padrão da frequência cardíaca (bpm) e da variação percentual nos diferentes tempos estudados em relação ao valor inicial

\begin{tabular}{|c|c|c|c|c|}
\hline Tempo (min) & $\begin{array}{c}\text { Média } \pm \\
\text { DP }\end{array}$ & $\begin{array}{c}\text { Variação } \\
(\%) \\
\text { Média } \pm \\
\text { DP }\end{array}$ & $\mathrm{n}$ & $p$ \\
\hline Inicial & $70,7 \pm 10,5$ & & 42 & \\
\hline Anestesia & $72,4 \pm 11,5$ & $3,1 \pm 14,1$ & 42 & 0,5829 \\
\hline 5 & $66,9 \pm 10,4$ & $-4,8 \pm 11,9$ & 42 & $0,0047^{*}$ \\
\hline 10 & $69,5 \pm 9,6$ & $-0,9 \pm 11,4$ & 42 & 0,3042 \\
\hline 15 & $67,6 \pm 9,5$ & $-2,4 \pm 14,6$ & 40 & $0,0038^{*}$ \\
\hline 20 & $68,1 \pm 9,1$ & $-0,9 \pm 13,8$ & 39 & 0,3788 \\
\hline 25 & $68,6 \pm 9,3$ & $-0,7 \pm 12,2$ & 38 & 0,4537 \\
\hline 30 & $68,6 \pm 9,0$ & $-1,4 \pm 13,8$ & 36 & 0,2964 \\
\hline 35 & $67,3 \pm 8,3$ & $-1,6 \pm 11,3$ & 29 & 0,2525 \\
\hline 40 & $69,2 \pm 9,6$ & $-1,3 \pm 8,9$ & 26 & 0,3926 \\
\hline 45 & $68,0 \pm 7,8$ & $-3,9 \pm 10,9$ & 22 & 0,0697 \\
\hline 50 & $71,9 \pm 8,8$ & $1,6 \pm 19,3$ & 15 & 0,9834 \\
\hline 55 & $75,3 \pm 12,5$ & $3,1 \pm 12,8$ & 12 & 0,5401 \\
\hline 60 & $72,7 \pm 9,2$ & $0,4 \pm 16,1$ & 12 & 0,8018 \\
\hline
\end{tabular}

teste t de Student pareado; teste de Wilcoxon pareado; DP - desvio padrão

A análise dos valores médios da pressão arterial média nos diferentes tempos estudados em relação ao valor inicial mostra diferença significativa aos 5, 10 15, 20, 30, 35, 40 e 45 minutos após a anestesia. Observou-se que a variação percentual média não ultrapassou $15 \%$ em relação ao valor inicial (Tabela 3 ). A análise individual dos casos mostrou que em 23 pacientes $(54,7 \%)$, pelo menos em algum momento dentre os 60 minutos, ocorreu uma variação acima de $20 \%$ da pressão arterial média (dados não mostrados).

A análise dos valores médios da frequência respiratória nos diferentes tempos estudados em relação ao valor inicial mostra diferença significativa em quase todos os tempos de avaliação, exceto aos 55 e 60 minutos após a anestesia. Observou-se que a variação percentual média não ultrapassou $15 \%$ em relação ao valor inicial (Tabela 4). A análise individual dos casos mostrou que em 41 pacientes $(97,6 \%)$, pelo menos em algum momento dentre os 60 minutos, ocorreu uma variação acima de $20 \%$ da frequência respiratória (dados não mostrados). 
Tabela 3-Valores médios e desvios padrões da pressão arterial média $(\mathrm{mmHg})$ e da variação percentual nos diferentes tempos estudados em relação ao valor inicial.

\begin{tabular}{ccccc}
\hline \hline Tempo (min) & Média \pm DP & $\begin{array}{c}\text { Variação (\%) } \\
\text { Média } \pm \text { DP }\end{array}$ & $\mathrm{n}$ & $\mathrm{p}$ \\
\hline \hline Inicial & $92,0 \pm 10,7$ & & 42 & \\
Anestesia & $95,4 \pm 14,7$ & $8,8 \pm 16,5$ & 42 & 0,1055 \\
5 & $99,3 \pm 14,9$ & $11,0 \pm 16,4$ & 42 & $0,0023^{*}$ \\
10 & $101,6 \pm 13,1$ & $8,2 \pm 13,9$ & 42 & $0,0001^{*}$ \\
15 & $98,7 \pm 12,1$ & $7,0 \pm 13,4$ & 40 & $0,0010^{*}$ \\
20 & $97,7 \pm 11,9$ & $4,8 \pm 15,3$ & 39 & $0,0041^{*}$ \\
25 & $96,2 \pm 14,0$ & $7,4 \pm 16,5$ & 38 & 0,0635 \\
30 & $94,4 \pm 13,7$ & $6,7 \pm 13,6$ & 36 & $0,0197^{*}$ \\
35 & $97,4 \pm 12,6$ & $7,6 \pm 13,1$ & 29 & $0,0233^{*}$ \\
40 & $98,5 \pm 12,7$ & $8,4 \pm 15,2$ & 26 & $0,0095^{*}$ \\
45 & $100,3 \pm 16,0$ & $4,5 \pm 13,5$ & 22 & $0,0212^{*}$ \\
50 & $97,5 \pm 13,1$ & $4,4 \pm 16,1$ & 15 & 0,2890 \\
55 & $98,1 \pm 13,7$ & $8,4 \pm 16,4$ & 12 & 0,5125 \\
60 & $101,8 \pm 12,6$ & $8,9 \pm 17,9$ & 12 & 0,1425 \\
\hline \hline
\end{tabular}

teste t de Student pareado; teste de Wilcoxon pareado; DP - desvio padrão

Tabela 4- Valores médios e desvios padrão da frequência respiratória (rpm) e da variação percentual nos diferentes tempos estudados em relação ao valor inicial

\begin{tabular}{ccccc}
\hline \hline Tempo (min) & Média \pm DP & $\begin{array}{c}\text { Variação (\%) } \\
\text { Média } \pm \mathrm{DP}\end{array}$ & $\mathrm{n}$ & $\mathrm{p}$ \\
\hline \hline Inicial & $12,5 \pm 5,2$ & & 42 & \\
Anestesia & $14,7 \pm 6,0$ & $38,1 \pm 85,9$ & 42 & $0,0461^{*}$ \\
5 & $19,3 \pm 12,0$ & $76,5 \pm 109,2$ & 42 & $0,0001^{*}$ \\
10 & $17,0 \pm 6,9$ & $61,6 \pm 105,8$ & 42 & $0,0006^{*}$ \\
15 & $15,7 \pm 6,2$ & $44,6 \pm 70,2$ & 40 & $0,0013^{*}$ \\
20 & $15,6 \pm 6,6$ & $45,5 \pm 73,2$ & 39 & $0,0014^{*}$ \\
25 & $14,7 \pm 5,5$ & $46,3 \pm 89,3$ & 38 & $0,0192^{*}$ \\
30 & $15,5 \pm 5,2$ & $51,3 \pm 74,0$ & 36 & $0,0023^{*}$ \\
35 & $15,9 \pm 6,1$ & $50,2 \pm 85,9$ & 29 & $0,0029^{*}$ \\
40 & $15,3 \pm 4,8$ & $45,7 \pm 83,8$ & 26 & $0,0127^{*}$ \\
45 & $15,3 \pm 4,3$ & $44,6 \pm 64,6$ & 22 & $0,0282^{*}$ \\
50 & $13,8 \pm 3,9$ & $43,9 \pm 68,0$ & 15 & $0,0453^{*}$ \\
55 & $14,3 \pm 3,9$ & $46,1 \pm 106,1$ & 12 & 0,3353 \\
60 & $13,3 \pm 3,9$ & $41,7 \pm 108,6$ & 12 & 0,7021 \\
\hline \hline
\end{tabular}

teste t de Student pareado; teste de Wilcoxon pareado; DP - desvio padrão

Os valores médios da saturação periférica de $\mathrm{O}_{2}$ se mantiveram acima de $95 \%$ (saturação normal) e não apresentaram diferença significativa em relação ao momento inicial. Um paciente apresentou hipoxemia grave e necessitou de suporte de oxigênio com cateter nasal.

Trinta e quatro pacientes não referiram dor e oito relataram dor em algum momento do procedimento. Os escores de dor de acordo com escala análogo-visual e a frequência (\%) em que ocorreram são mostrados na Figura 1. Sete pacientes que referiram dor com pontuação maior do que seis, necessitaram de complementação com anestésico local. A dor foi referida principalmente nos momentos da osteotomia (5 pacientes), odontosecção (2 pacientes) e luxação (1 paciente).

Em relação à ansiedade avaliada no momento da admissão dos pacientes no consultório, observou-se que 22 pacientes encontravam-se calmos e 20 apresentavam algum grau de ansiedade (Figura 2).

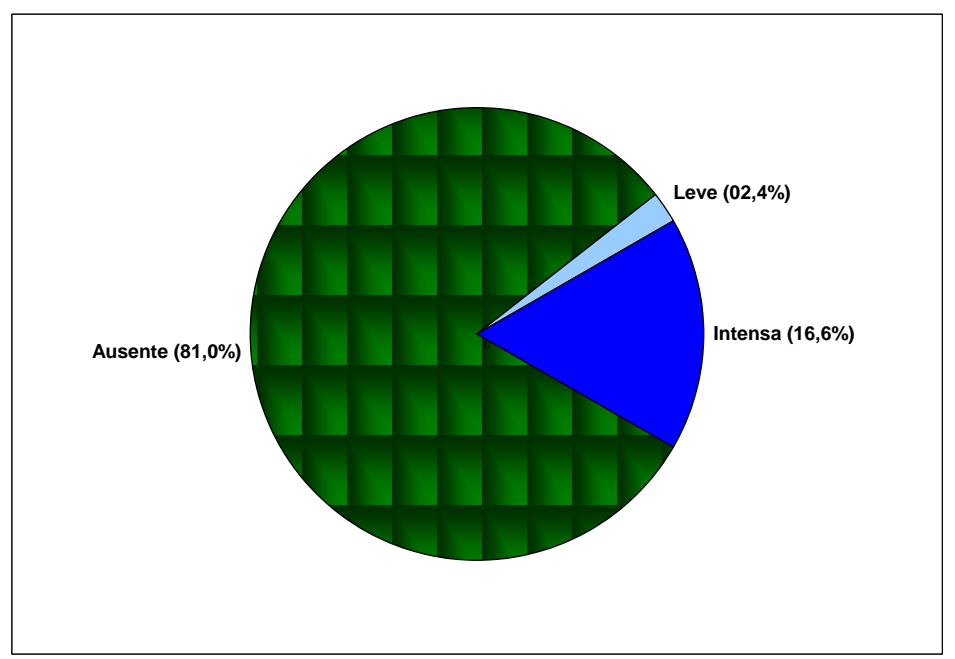

Figura 1- Distribuição percentual de pacientes de acordo com a intensidade da dor (EAV)

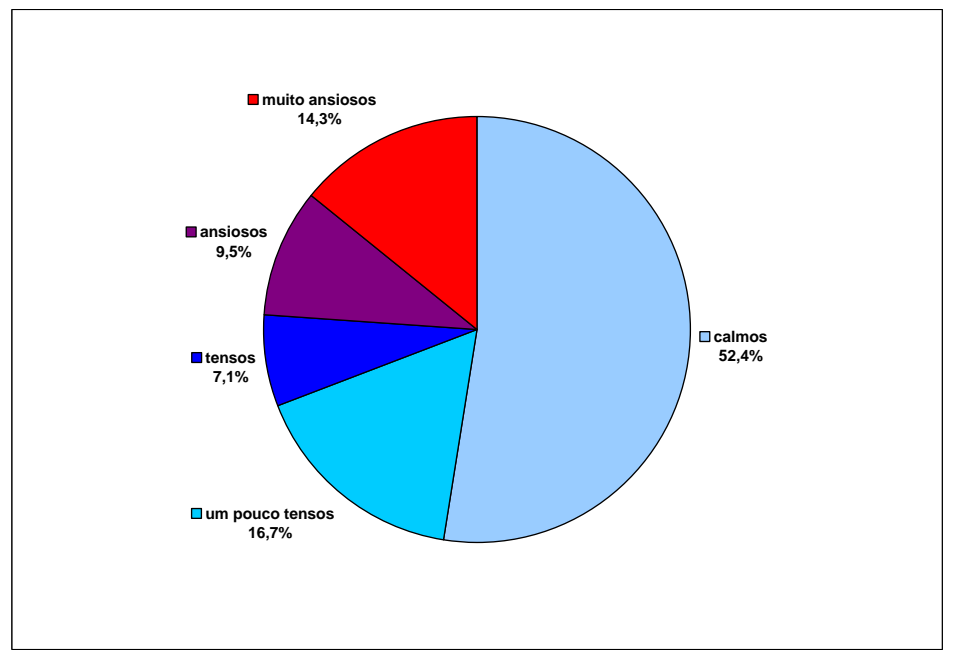

Figura 2- Distribuição percentual de pacientes de acordo com o Grau de Ansiedade 


\section{Discussão}

A monitorização de pacientes durante a extração cirúrgica de molares é oportuna, principalmente em procedimentos com previsão de grande traumatismo ou em pacientes cujas condições psicológicas tornem esse controle desejável para aumentar a segurança. A segurança do paciente constitui o principal objetivo da monitorização durante as cirurgias. Embora seja raramente utilizada em procedimentos odontológicos normais, ressalta-se que, apesar de raras, algumas situações emergenciais podem ocorrer, mesmo em pacientes saudáveis (LUOTIO; MATTILA; KOTILAINEN, 1993; ARRIGONI; LAMBRECTH; FILIPPI, 2005). A monitorização básica fornece informações sobre os principais sinais vitais,

cardiocirculatórios e respiratórios, particularmente pressão arterial, frequência e ritmo cardíacos, podendo ser complementada com oxímetro de pulso para avaliação da saturação de oxigênio e frequência respiratória (POLIDO; OLIVEIRA, 1998; ALEMANYMARTINEZ et al., 2008).

As alterações observadas na frequência cardíaca e pressão arterial média podem ser atribuídas aos vasoconstritores associados aos anestésicos locais normalmente usados durante a extração de molares, assim como aos outros fatores concomitantes tais como dor, ansiedade ou estresse do paciente (TOLAS; PFLUG; HALTER, 1982; BRAND; ABRAHAM-INPIJN, 1996; NICHOLS, 1997; TAKAHASHI et al., 2005).

Alguns autores relataram ausência de repercussões hemodinâmicas em pacientes sob efeitos de anestésico local com epinefrina, ao contrario do observado por outros que encontraram alterações da frequência cardíaca e da pressão arterial, sem, no entanto, relacionálas ao vasoconstritor (MUSYKA; CIGLICK, 1997; BÁGUENA; CHIVA, 1999). Nossos resultados vão ao encontro dos achados de ALEMANY-MARTINEZ et al.(2008), que observaram alterações desprezíveis da pressão arterial, em pacientes submetidos à remoção cirúrgica de terceiros molares inferiores sob anestesia com articaina associada à epinefrina. Essas alterações podem ocorrer principalmente durante a osteotomia, a odontosecção e a avulsão dentária, considerados os momentos de maior estresse do procedimento cirúrgico (PARAMAESVARAN; KINGON, 1994; SILVESTRE et al., 2001). Essas repercussões também podem ser evidenciadas no inicio do procedimento, e devem-se principalmente à liberação endógena de catecolaminas causada pela ansiedade ou medo, apresentados pelos pacientes durante a visita ao dentista (NICHOLS, 1997).

Com relação à frequência respiratória, foram observadas alterações significativas durante quase todo o período de avaliação, resultados semelhantes aos descritos por outros autores, que atribuíram essas alterações a emoções como dor, medo e ansiedade (MASAOKA; HOMMA , 1999; MASAOKA; HOMMA, 2001; MASAOKA et al., 2004).

O provável mecanismo para o aumento da frequência respiratória, seria a ativação do polo temporal e da amígdala do sistema límbico, por alterações emocionais ocasionadas por dor e ansiedade presentes neste tipo de procedimento (MASAOKA; HOMMA , 2000).

Em cada paciente, o grau de ansiedade foi avaliado antes do procedimento empregando-se uma escala validada e útil como preditor do estresse do paciente em relação ao tratamento dentário. O conhecimento do grau de ansiedade do paciente constitui ferramenta de grande importância para o cirurgião dentista, visto que possibilita que o mesmo fique atento em relação às respostas do paciente diante de situações de estresse e institua medidas que possam aliviar o grau de ansiedade e consequentemente diminuir as repercussões hemodinâmicas e respiratórias (ARRIGONI; LAMBRECTH; FILIPPI, 2005; FUKAYAMA; YAGIELA, 2006).

Neste estudo, vinte pacientes apresentavam algum grau de ansiedade, e vinte e dois relataram estar calmos. Essa pequena diferença entre pacientes ansiosos e não ansiosos foi também relatada em trabalho da literatura e atribuída ao baixo poder estatístico da amostra (ALEMANY-MARTINEZ et al.,2008).

É evidente que a associação de estresse e ansiedade com dor observada em cirurgias para extração de dentes molares, também induz à secreção de catecolaminas e consequentemente ocasionam intercorrências intra-operatórias. Dor intensa foi referida por sete pacientes nos momentos da osteotomia, odontosecção e luxação que necessitaram de complementação com anestésico local. Estudo anterior realizado para avaliar a percepção e as principais causas de dor durante extração dentária mostrou que o limiar de dor é menor em homens do que em mulheres (FAGADE; OGINNI, 2005), resultados similares aos de outros autores, que também observaram em homens menor tolerância à dor, mas em contrapartida apresentam maior capacidade para controlar a ansiedade (ALEMANY-MARTINEZ et al., 2008). Ao contrário do acima descrito, tem sido relatado na literatura que os homens referem menos dor, durante procedimentos cirúrgicos (COLORADO-BONNIN et al., 2006).

A monitorização, por possibilitar detecção fidedigna de repercussões intraoperatórias, adquire grande importância em pacientes com perfil psíquico ou portadores de doenças sistêmicas susceptíveis à descompensação sob intenso estresse. Nesse grupo de pacientes, a provisão de ansiólise ou sedação permite a realização de procedimentos cirúrgicos com o conforto desejado e mínima alteração autonômica, refletindo-se num curso intraoperatório mais estável e seguro (POLIDO; OLIVEIRA, 1998; GEMAL; DURAN, 2007).

\section{Conclusão}

Os resultados deste estudo permitem concluir que alterações hemodinâmicas e respiratórias podem ocorrer durante extrações de terceiros molares retidos. A monitorização eletrônica possibilita ao cirurgião-dentista diagnosticar precocemente essas alterações, e consequentemente diminuir a morbi-mortalidade nesse tipo de procedimento.

\section{Referências}

ALEMANY-MARTINEZ, A. et al. Hemodynamic changes during the surgical removal of lower third molar. J. Oral Maxillofac. Surg., Philadelphia, v. 66, no. 3, p. 453-461, Mar. 2008.

ARRIGONI, J.; LAMBRECTH, J.T.; FILIPPI, A. Cardiovascular monitoring and its consequences in oral surgery. Schweiz Monatsschr Zahnmed, Bern, v. 115, no. 3, p. 208-213, 2005.

BÁGUENA, J.C.; CHIVA, F. Efectos de los anestésicos de uso odontológico sobre la presión arterial y la frecuencia cardíaca. Rev. Eur. Odontoestomatol., [S.I.], no. 5, p. 291-295, 1999.

BRAND, H.S. et al. Cardiovascular and neuroendocrine response during acute stress induced by different types of dental treatment. Int. Dent. J., London, v. 45, no. 1, p. 45-48, Feb. 1995.

BRAND, H.S.; ABRAHAM-INPIJN, L. Cardiovascular responses induced by dental treatment. Eur. J. Oral Sci., Copenhagen, v. 104, no. 3, p. 245-252, June 1996.

BRAZ, J.R.C. Monitorização da oxigenação e da ventilação. Rev. Bras. Anestesiol., Rio de Janeiro, v. 43, n. 3, p. 223-240, jun. 1996.

Rev. Fac. Odontol. Porto Alegre, v. 51, n. 2, p. 9-14, mai./ago., 2010. 
COLORADO-BONNIN, M. et al. Quality of life following lower third molar renoval. Int. J. Oral Maxillofac. Surg., Philadelphia, v. 35, no. 4, p. 343-347, 2006.

CORAH, N.L.; GALE, E.N.; ILLIG, S.J. Assessment of a dental anxiety scale. J. Am. Dental. Assoc., Chicago, v. 97, no. 5, p. 816-819, 1978.

CROSWELL, R.J.R. et al. A comparison of conventional versus electronic monitoring of sedated pediatric dental patients. Pediatr. Dent., Chicago, v. 17, no. 5, p. 332-339, 1995.

FAGADE, O.O.; OGINNI, F.O. Intraoperative pain perception in tooth extraction: Possibles causes. Int. Dent. J., London, v. 55, no. 4, p. 242-246, Aug. 2005.

FUKAYAMA, H.; YAGIELA, J.A. Monitoring of vital signs during dental care. Int. Dent. J., London, v. 56, no. 2, p. 102-108, Apr. 2006.

GEMAL, A.E.; DURAN, M.S. Anestesia para procedimentos em odontologia geral. In: CAVALCANTI, I.L.; ASSAD, A.R.; LACERDA, M.A. Anestesia fora do centro cirúrgico. Rio de Janeiro: Sociedade de Anestesiologia do Estado do Rio de Janeiro, 2007. p. 267-282.

LUOTIO, K.; MATTILA, M.A.; KOTILAINEN, R.M. Introduction a new patient monitoring system during dental procedures: pulse oximetry. Oral Surg. Oral Diagn., Kuopio, Finland, v. 4, p. 29-33,1993.

MASAOKA, Y.; HOMMA, I. Expiratory time determinated by individual anxiety levels in humans. J. Appl. Physiol., Washington, v. 86, no. 4, p. 1329-1336, Apr. 1999.

The effect of anticipatory anxiety on breathing and metabolism in humans. Respir. Physiol., Amsterdam, v. 128, no. 2, p. 171-177, Nov. 2001.

The source generator of respiratory-related anxiety potential in the human brain. Neurosc. Lett., Amsterdam, v. 283, no. 1, p. 21-24, Mar. 2000.

MASAOKA, Y. et al. Breathing patterns associated with trait anxiety and breathlessness in humans. Jpn. J. Physiol., Tokyo, v. 54, no. 5, p. 465-470, Oct. 2004.

MUSYKA, B.C.; CIGLICK, M. The hypertensive dental patient. J. Am. Dent. Assoc., Chicago, v.128, no. 8, p. 1109-1120, Aug. 1997.

NICHOLS, C. Dentistry and hypertension. J. Am. Dent. Assoc., Chicago, v. 128, no.11, p. 1557-1562, Nov. 1997.

PARAMAESVARAN, M.; KINGON, A.M. Alterations in blood pressure and pulse rate in exodontia patients. Aust. Dent. J., Sidney, v. 39, no. 5, p. 282-286, Oct. 1994.

POLIDO, W.; OLIVEIRA, F.A.M. Uso do oxímetro de pulso em odontologia. Rev. Gauch. Odontol., Porto Alegre, v. 46, n. 4, p. 197200, out./dez. 1998.

SILVESTRE, F.J. et al. Efectos de los vasoconstrictores usados en odontología sobre la presión arterial sistólica y diastólica. Med. Oral, Madrid, v.6, no. 1, p. 57-63, ene. 2001.

TAKAHASHI, T. et al. The effects of epinephrine in local anesthetics on plasma catecholamine and hemodynamic responses. Odontology, [S.I.], v. 93, no. 1, p. 72-79, Sept. 2005.

TOLAS, A.G.; PFLUG, A.E.; HALTER, J.B. Arterial plasma epinephrine concentration and hemodynamic response after dental injection of local anesthesia plus epinephrine. J. Am. Dent. Assoc., Chicago, v. 104, no.1, p. 41-43, Jan. 1982. 